\title{
Capacitor Siting Using Benders Decomposition
}

\author{
Zhixin Miao, Senior Member, IEEE, Lakshan Piyasinghe, Student Member, IEEE, Lingling Fan, Senior \\ Member, IEEE
}

\begin{abstract}
This paper presents an optimization problem of siting capacitors in a power network to achieve economic benefits. The formulated optimization problem is a nonlinear mixed integer programming problem. In order to find an approximate optimal solution, Benders decomposition is adopted. The optimization problem will be decomposed into a main problem and a subproblem. The subproblem determines dispatch patterns for generators to minimize an objective function if the sites of the capacitors are given. The subproblem is a conventional AC optimal power flow problem. Existing toolboxes such as MATPOWER is used to solve such problems. The subproblem helps to create Benders cuts for the main problem which decides where to put capacitors with a set of cuts introduced accumulatively from the subproblems at each iteration. The main problem is solved by MATLAB CVX toolbox. Numerical results for IEEE 118-bus system are given.
\end{abstract}

\section{INTRODUCTION}

Give a set of capacitors, where to site them to achieve the best economic benefit is a nonlinear mixed integer programming problem. Back in 1989, this problem was already described in [1]. The strategy of problem solving is Benders decomposition where the entire optimization problem is decomposed into two problems. One problem focuses on the conventional optimal dispatch problem while the other focuses on mixed integer programming problem. In that paper, the authors explained the modeling details. Benders decomposition solving strategy only shows up in the appendix.

Benders decomposition has been adopted to solve similar type of mixed integer nonlinear programming problems. For example, in [2], [3], a unit commitment problem with $\mathrm{AC}$ optimal power flow (OPF) is solved by Benders decomposition. Once the unit on/off is decided, for each hour, an AC OPF problem is solved. This subproblem introduces cuts to the main problem. And the main problem looks at unit scheduling with the constraints introduced by the Benders cuts. Benders cuts replace a set of nonlinear constraints. The main problem becomes a mixed integer linear programming problem.

More recently, Benders decomposition has been adopted in stochastic programming [4] and robust programming [5] to handle scenarios of large dimensions. For example, in [4], a stochastic unit commitment problem considering wind variation is to be solved. The wind variation is represented by various scenarios with probability assigned. Given the unit on/off schedules, for each scenario, an AC OPF problem will be solved and Benders cuts will be constructed. In the main problem, unit on/off decision will be made based on the temporal constraints such as ramp up/down limits and the

Z. Miao, L. Piyasinghe, and L. Fan are with Department of Electrical Engineering at University of South Florida, Tampa, FL 33620 (Emails: zmiao@usf.edu.)
Benders cuts from the subproblems. Using this decomposition technique, large-scale stochastic programming problem can now be solved.

The objective of this paper is to adopt Benders decomposition method to site fixed size capacitors. Unlike [1], we focus on the mathematics of Benders decomposition in capacitor siting problems. In addition, our research adopts the toolboxes introduced after 1989 in the research community: MATPOWER [6] for AC OPF, CVX [7] (a MATLAB toolbox for convex optimization using solvers such as GUROBI) and GUROBI [8] ( a solver for mixed integer programming problem solving).

The rest of the paper is organized as follows. Section II gives the capacitor siting mathematical programming problem formulation. Section III gives the details on Benders decomposition application in capacitor siting. Section IV presents the numerical study results. Section V concludes the paper.

\section{Mathematical Programming Formulation}

Capacitor siting while considering an AC OPF is formulated as follows. The capacitors are assumed to have fixed size $Q_{c}$. A binary variable $u_{i}$ indicates if Bus $i$ will have capacitor installed or not.

$$
\begin{array}{r}
\text { min } \\
\text { s.t. } \\
P_{g}-P_{L}-P(V, \theta)=0 \\
Q_{g}-Q_{L}-Q(V, \theta)=0 \\
u_{i} Q_{c}=Q_{i}(V, \theta) \\
S(V, \theta)-S^{M} \leq 0 \\
V^{m} \leq V \leq V^{M} \\
P_{g}^{m} \leq P_{g} \leq P_{g}^{M} \\
Q_{g}^{m} \leq Q_{g} \leq Q_{g}^{M} \\
\sum_{i} u_{i}=N_{c}
\end{array}
$$

where

$f($.$) is the cost function, superscript M$ denotes upper bound and $m$ denotes low bound.

$P_{g}, Q_{g}, P_{L}$ and $Q_{L}$ are the vectors of bus real and reactive power injection, and real and reactive loads.

$P(V, \theta)$ and $Q(V, \theta)$ are the power injection expressions in terms of bus voltage magnitude and phase angles.

$S(V, \theta)$ is the vector of line complex power flow.

$u_{i}$ is a binary variable. $u_{i}=1$ indicates a capacitor is installed at Bus i. $u_{i}=0$ means no capacitor installed.

The two equality constraints are power flow equations. The first inequality constraint denotes the line flow limit. The rest three inequalities denote the voltage requirement and generator exporting power limits. 


\section{BENDERS DECOMPOSITION}

Benders decomposition is applied to the above nonlinear mixed integer programming problem. The binary variables $u$ will be separated from the rest of the continuous decision variables such as $P_{g}, Q_{g}, V$ and $\theta$. When the binary variable vector $u$ is fixed, or the sites of capacitor installation are given, how to dispatch generators to achieve economic is a conventional AC OPF problem. Based on the current vector $u$, a cutting plane can be constructed. Benders cuts are dual cuts.

Subproblem:

$$
\begin{array}{r}
\text { min } \\
f\left(P_{g}\right) \\
\text { s.t. } \\
P_{g}-P_{L}-P(V, \theta)=0 \\
Q_{g}-Q_{L}-Q(V, \theta)=0 \\
\hat{u}_{i} Q_{c}=Q_{i}(V, \theta) \\
S(V, \theta)-S^{M} \leq 0 \\
V^{m} \leq V \leq V^{M} \\
P_{g}^{m} \leq P_{g} \leq P_{g}^{M} \\
Q_{g}^{m} \leq Q_{g} \leq Q_{g}^{M}
\end{array}
$$

Let the constraints related to vector $u$ be relaxed. Examine the partial dual problem of the original problem, we have:

$$
\begin{array}{cc}
\max _{\lambda} & \min f\left(P_{g}\right)+\sum_{i} \lambda_{i}\left(Q_{i}(V, \theta)-u_{i} Q_{c}\right) \\
\text { s.t. } P_{g}-P_{L}-P(V, \theta)=0 \\
Q_{g}-Q_{L}-Q(V, \theta)=0 \\
S(V, \theta)-S^{M} \leq 0 \\
V^{m} \leq V \leq V^{M} \\
P_{g}^{m} \leq P_{g} \leq P_{g}^{M} \\
Q_{g}^{m} \leq Q_{g} \leq Q_{g}^{M}
\end{array}
$$

For a given vector $\hat{u}$ (feasible), solving Problem (1) leads to solutions to all decision variables. Since the partial dual problem is a maximizing problem where the optimal solution should always be greater or equal to that corresponds to any given $\hat{u}$, therefore, the cutting plane can be constructed based on the partial dual as:

$$
\eta \geq f\left(\hat{P}_{g}\right)+\sum_{i} \hat{\lambda}_{i}\left(\hat{Q}_{i}-u_{i} Q_{c}\right)
$$

Note that $\hat{Q}_{i}=\hat{u}_{i} Q_{c}$, and $f\left(\hat{P}_{g}\right)$ is the cost function computed based on $\hat{u}$. The above cut can rewritten as:

$$
\eta \geq \hat{\text { ost }}+\sum_{i} \hat{\lambda}_{i} Q_{c}\left(\hat{u}_{i}-u_{i}\right)
$$

The cut can also be viewed as subgradient based update for the decision variables $u_{i}$. For the maximization problem, the optimal $u_{i}$ should make the final cost greater or equal than cost $+\sum_{i} \hat{\lambda}_{i} Q_{c}\left(\hat{u}_{i}-u_{i}\right)$.

This cut will be used for the master problem of capacitor switching. Suppose there are $K$-step iterations, then there will be $K$ cuts.

$$
\begin{array}{r}
\min _{\eta, u} \\
\text { s.t. } \quad \eta \geq \hat{\operatorname{cost}}^{(k)}+\sum_{i}{\hat{\lambda_{i}}}^{(k)} Q_{c}\left(\hat{u}_{i}^{(k)}-u_{i}\right) \\
k=1, \cdots, K \\
\sum_{i} u_{i}=N_{c}
\end{array}
$$

The above problem is a mixed integer linear programming problem and can be solved by existing toolboxes such as CVX [7].

Values from the master problems are the lower bounds of the original problem since partial dual problems are introduced. Values from the sub problems are the upper bounds of the original problem since $u$ is a vector inside the feasible region and the problem gives a feasible solution. When the gap between the lower bound and upper bound is less than a threshold, the iteration process can be terminated and a solution is found.

\section{CAse Studies}

IEEE 118-bus system (Fig. 1) is selected for case study. Total there are 64 load buses. These buses are candidates for installing capacitors. The size of each capacitor is assumed

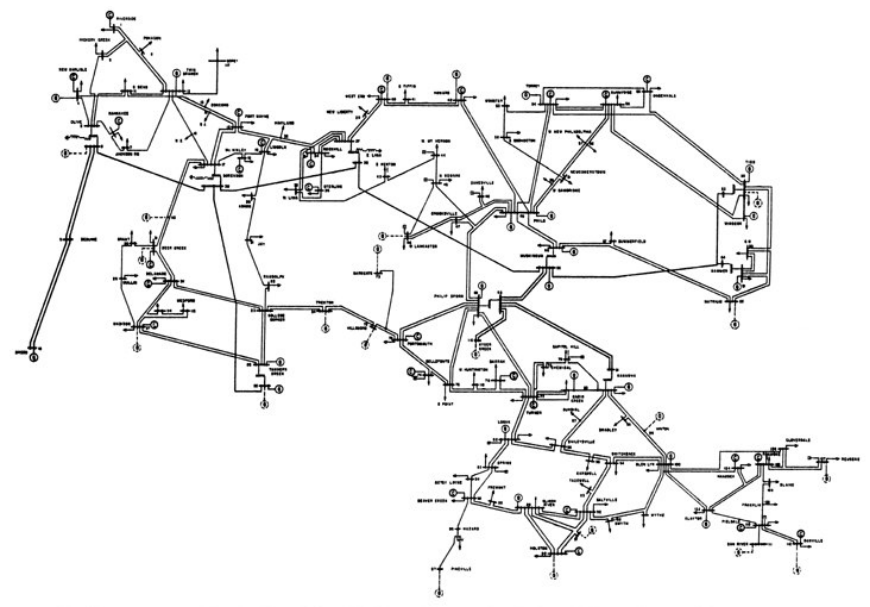

Fig. 1. IEEE 118-bus system [9].

to be 5 MVar. MATLAB codes were developed for this problem. The main problem is solved by CVX toolbox with GUROBI solver while the subproblem (AC OPF) is solved MATPOWER. Reactive MATPOWER gives reactive power prices at all nodes. These prices will be used to generate cuts for the main problem. The following total numbers to be installed are checked: 5, 10, 15, 20 and 30. For each case, the values of the main problem and the subproblem at each iteration are plotted.

Since the AC OPF is a nonconvex optimization problem, the results obtained need examination for their optimality.

Fig. 2 gives the results when five capacitors are planned. It is shown that the values of the main problem and the subproblem converge. At iteration 2, it is found that the value of the main problem is lower than the values at iteration 9. Solution to the 


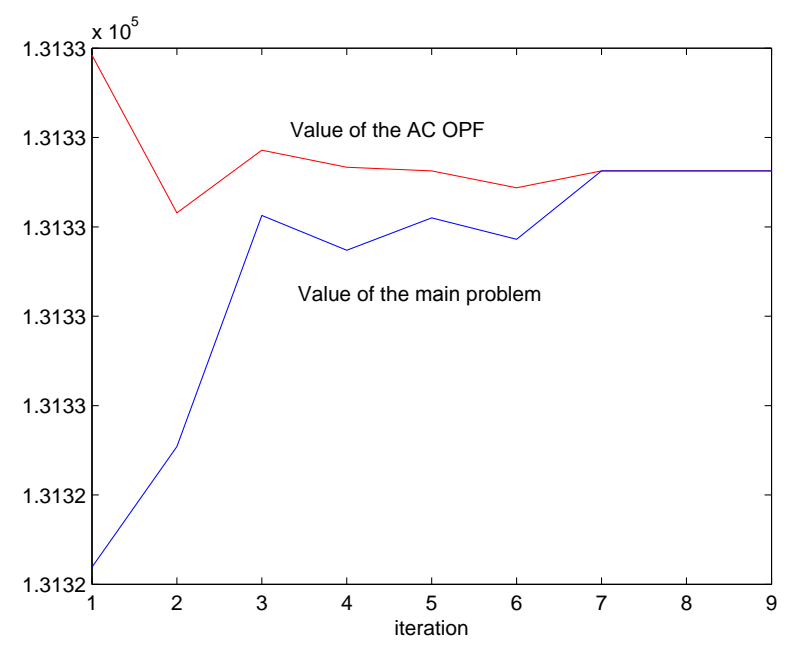

Fig. 2. Total number of capacitors: 5 .

AC OPF is a feasible solution. Therefore, the siting should be based on the decision variables from iteration 2 .

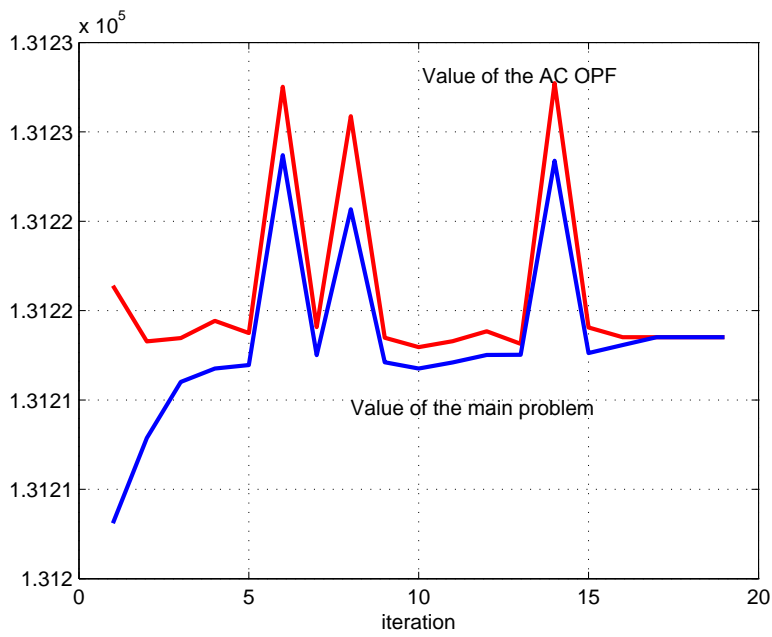

Fig. 3. Total number of capacitors: 10 .

Fig. 3 gives the results when 10 capacitors are planned. It is shown that the values of the main problem and the subproblem converge after iteration 18 . At iteration 10 , it is found that the value of the main problem is lower than the values at iteration 18. Solution to the AC OPF is a feasible solution. Therefore, the siting should be based on the decision variables from iteration 10.

Fig. 4 gives the results when 15 capacitors are planned. It is shown that the values of the main problem and the subproblem converge after iteration 12. At iteration 3 , it is found that the value of the main problem is lower than the values at iteration 12. Solution to the AC OPF is a feasible solution. Therefore, the siting should be based on the decision variables from iteration 3.

Fig. 5 gives the results when 20 capacitors are planned. It is shown that the values of the main problem and the subproblem converge after iteration 6 . At iteration 4 , it is found that the

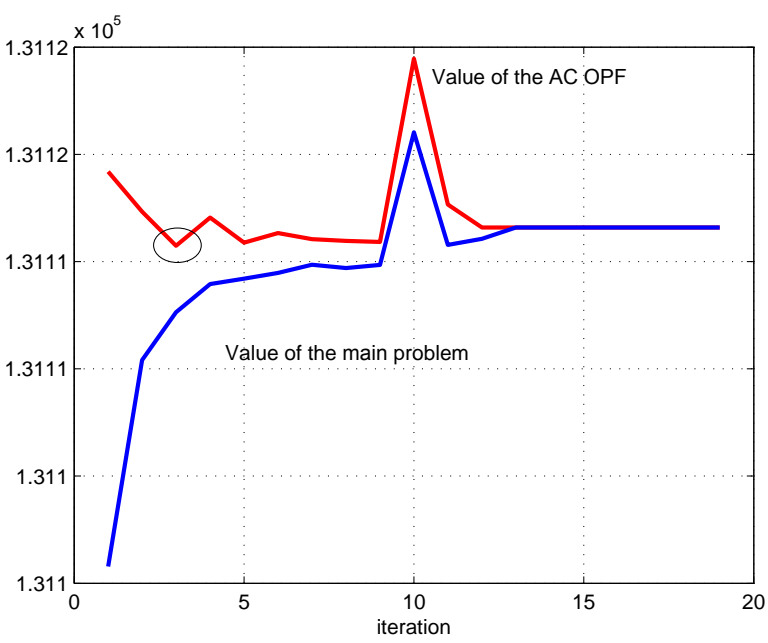

Fig. 4. Total number of capacitors: 15 .

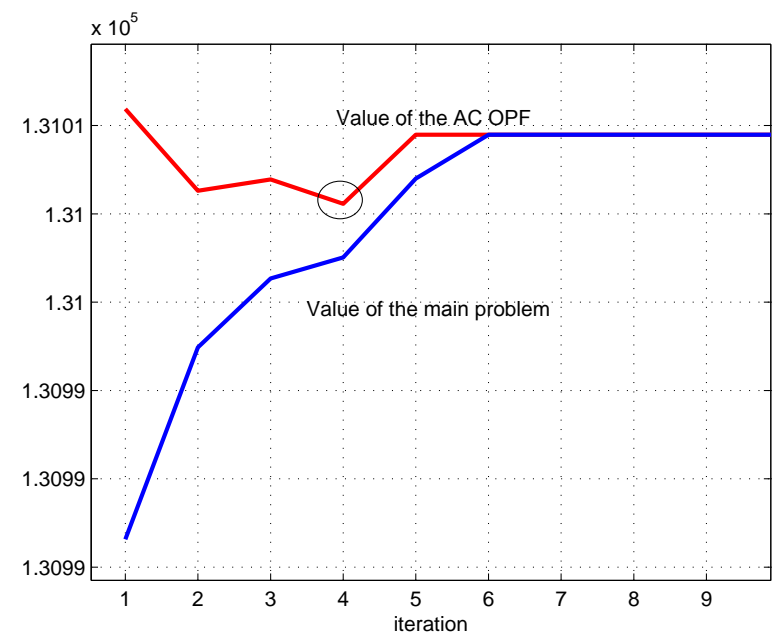

Fig. 5. Total number of capacitors: 20 .

value of the main problem is lower than the values at iteration 6. Solution to the AC OPF is a feasible solution. Therefore, the siting should be based on the decision variables from iteration 4.

Fig. 6 gives the results when 30 capacitors are planned. It is shown that the values of the main problem and the subproblem converge after iteration 4. And this value is the minimum among all values from the AC OPF and the maximum of all values from the main problem. Therefore, the siting should be based on the decision variables of the converged results at iteration 4-9.

Fig. 7 presents the bus locations for the capacitors for the five scenarios.

Remarks: The case study is carried out in IEEE-118 system. It is well-known that $\mathrm{AC} \mathrm{OPF}$ is a nonconvext optimization problem. For non-convex problems, Benders decomposition may not work well. The case studies show that for four scenarios out of five, the converged solutions from Benders decomposition are not the optimal solution.

For each cast study, discretion has to be applied when 


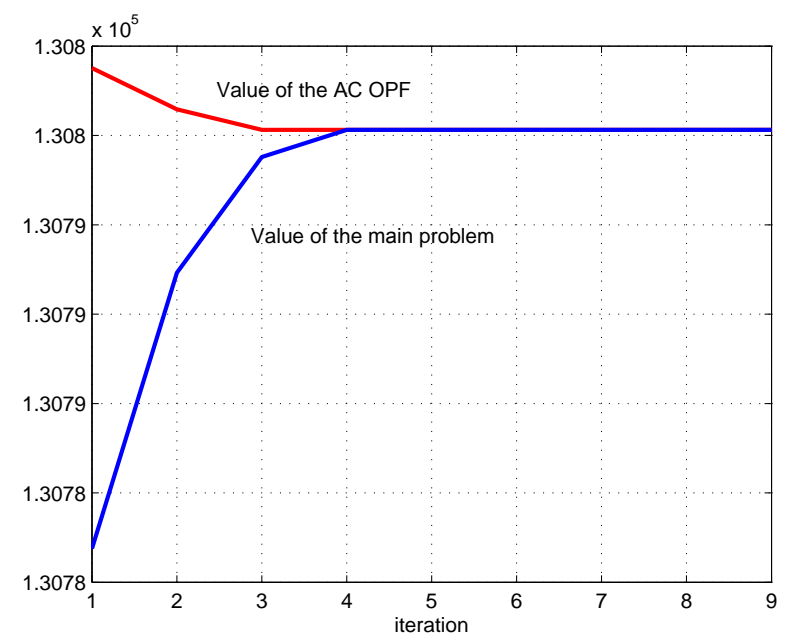

Fig. 6. Total number of capacitors: 30 .

\begin{tabular}{|r|r|r|r|r|}
\hline 5 caps & 10 caps & 15 caps & 20 caps & 30 caps \\
\hline & 1 & 1 & 1 & 1 \\
\hline & & & & 2 \\
\hline 7 & & & & 6 \\
\hline & & 7 & 7 & 7 \\
\hline & & & & 9 \\
\hline & & & 11 & 11 \\
\hline & & 12 & & 12 \\
\hline & & & & 13 \\
\hline & & 15 & 15 & 15 \\
\hline 16 & 16 & 16 & 16 & 16 \\
\hline & & & 18 & 18 \\
\hline & & & 22 & 22 \\
\hline & 23 & 23 & 23 & 23 \\
\hline & & & & 30 \\
\hline & 31 & 31 & 31 & 31 \\
\hline 32 & 32 & 32 & 32 & 32 \\
\hline & & 34 & 34 & 34 \\
\hline & & & & 47 \\
\hline & & & 48 & 48 \\
\hline & & & 50 & 50 \\
\hline & & 51 & 51 & 51 \\
\hline 52 & 52 & 52 & 52 & 52 \\
\hline & & & & 53 \\
\hline & 56 & 56 & 56 & 56 \\
\hline & 58 & 58 & 58 & 58 \\
\hline & & & 59 & 59 \\
\hline & & 60 & 60 & 60 \\
\hline 63 & 63 & 63 & 63 & 63 \\
\hline & & & & 64 \\
\hline & & & & \\
\hline
\end{tabular}

Fig. 7. Capacitor installation sites. interpreting the results. In this type of mixed integer nonconvex programming applications, Benders decomposition is very useful to provide approximately optimal solutions.

\section{CONCLUSiON}

In this paper, a capacitor siting problem is solved using Benders decomposition. The binary variables determining capacitor siting are separated from the differentiable variables for AC OPF. Given the set of the binary decision variables, AC OPF will be solved and Benders cuts are constructed. The main problem determines the binary decision variables based on the Benders cuts. An IEEE-118 bus system is used to test the algorithm of siting. The study results demonstrate how to interpret results from Benders decomposition for nonconvex optimization problems.

\section{ACKNOWLEDGEMENT}

The authors would like to acknowledge Dr. Bo Zeng for his suggestion on Benders decomposition.

\section{REFERENCES}

[1] M. E. Baran and F. F. Wu, "Optimal capacitor placement on radial distribution systems," Power Delivery, IEEE Transactions on, vol. 4, no. 1, pp. 725-734, 1989.

[2] N. Alguacil and A. Conejo, "Multiperiod optimal power flow using benders decomposition," Power Systems, IEEE Transactions on, vol. 15, no. 1, pp. 196-201, 2000.

[3] H. Ma and S. Shahidehpour, "Decomposition approach to unit commitment with reactive constraints," in Generation, Transmission and Distribution, IEE Proceedings-, vol. 144, no. 2. IET, 1997, pp. 113-117.

[4] J. Wang, M. Shahidehpour, and Z. Li, "Security-constrained unit commitment with volatile wind power generation," Power Systems, IEEE Transactions on, vol. 23, no. 3, pp. 1319-1327, 2008.

[5] D. Bertsimas, E. Litvinov, X. A. Sun, J. Zhao, and T. Zheng, "Adaptive robust optimization for the security constrained unit commitment problem,” Power Systems, IEEE Transactions on, vol. 28, no. 1, pp. 52-63, 2013.

[6] R. D. Zimmerman, C. E. Murillo-Sánchez, and R. J. Thomas, "Matpower: Steady-state operations, planning, and analysis tools for power systems research and education," Power Systems, IEEE Transactions on, vol. 26, no. 1, pp. 12-19, 2011.

[7] CVX Research Inc., "CVX: Matlab software for disciplined convex programming, version 2.0 beta," http://cvxr.com/cvx. Sep. 2012.

[8] Gurobi, "Gurobi optimizer reference manual," URL: http://www. gurobi. com, 2012.

[9] R. Christie. (1993) Power systems test case archive. [Online]. Available: https://www.ee.washington.edu/research/pstca/pf118/pg_tca118bus.htm 